TITLE:

\title{
Spontaneous Generation of Giant Liposomes from an Oil/Water Interface
}

\section{$\operatorname{AUTHOR}(\mathrm{S}):$}

Yamada, Ayako; Le Berre, Maël; Yoshikawa, Kenichi; Baigl, Damien

\section{CITATION:}

Yamada, Ayako ... [et al]. Spontaneous Generation of Giant Liposomes from an Oil/Water Interface. Chembiochem 2007, 8(18): 2215-2218

\section{ISSUE DATE:}

2007-10

URL:

http://hdl.handle.net/2433/49731

\section{RIGHT:}

Copyright (c) 2007 WILEY-VCH Verlag GmbH \& Co. KGaA, Weinheim; This

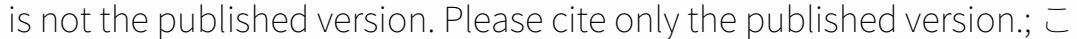
の論文は出版社版でありません。引用の際には出版社版をご確認ご利 用ください。 


\title{
Spontaneous Generation of Giant Liposomes from an Oil/Water Interface
}

\author{
Ayako Yamada, ${ }^{[\mathrm{b}]}$ Maël Le Berre, ${ }^{[\mathrm{a}]}$ Kenichi Yoshikawa, ${ }^{[\mathrm{b}, \mathrm{c}]}$ and Damien Baigl ${ }^{*[\mathrm{a}, \mathrm{c}]}$
}

[a] Department of Chemistry, Ecole Normale Sup érieure

[b] Department of Physics, Graduate School of Science, Kyoto University

[c] Spatio-Temporal Order Project, ICORP, JST

Keywords: biomimetics, liposomes, oil/water interface, phospholipids, self-assembly

Giant liposomes, phospholipid vesicles with a bilayered membrane and a size greater than $1 \mu \mathrm{m}$, have attracted a strong scientific interest mainly motivated by trying to synthesize artificial cells.[1] Indeed, giant liposomes have been shown to be the best primary model of cells in terms of size, membrane composition, or the ability to encapsulate biologically relevant molecules.[1b, 2] Hence, various methodologies for the preparation of cell-sized liposomes have been proposed, such as natural swelling,[3] freezing and thawing,[4] electroformation,[5] extraction from a cell membrane,[6] transfer of microdroplets,[7] and microfluidic methods.[8] Among them, the widely used natural swelling method, in which liposomes generate from a dry lipid film, is usually described as a spontaneous phenomenon, and its mechanism has been investigated.[9] Here, we report a new spontaneous phenomenon in a biphasic (oil/water) environment that leads to the formation of giant liposomes with a diameter ranging from 1 to $100 \mu \mathrm{m}$. We observed that giant liposomes spontaneously formed when a horizontal liquid interface was made between an upper oil phase containing phospholipid and a lower water phase. Our method mainly differs from natural swelling in that liposomes are obtained from a liquid oil/water interface at which the lipid organization and dynamics differ from that on a usual solid substrate. In this study, we used DOPC phospholipid dissolved in mineral oil at various concentrations, and characterized the peculiar phospholipid organization at the interface as well as the obtained giant liposomes by confocal microscopy. 
An oil/water interface was prepared in a cylindrical observation chamber made of poly(dimethyloxane) (PDMS) walls bound to a glass microscope cover slide (Figure 1). A thin layer of water was placed at the bottom of the observation chamber, which was topped up with a thin layer of mineral oil containing 1,2-dioleoyl-sn-glycero-3-phosphocholine (DOPC) phospholipid and a small fraction of 1-oleoyl-2-[12-[(7-nitro-2-1,3-benzoxadiazol-4-yl)amino]dodecanoyl]-sn-glycero-3-pho sphocholine (NBD-PC) fluorescent phospholipid.

First, the experiment was performed with a DOPC concentration of $1 \mu \mathrm{m}$ in oil and a temperature of $25{ }^{\circ} \mathrm{C}$. One minute after interface preparation, we observed the formation of small phospholipid aggregates randomly distributed at the oil/water interface, which presented some irregularities. (Figure 2A top) The presence of liposomes was not detected in either phase. With time, the interface became more indented as phospholipid aggregates slowly reorganized. $18 \mathrm{~h}$ after interface preparation, phospholipid molecules were arranged into a dotted pattern at the interface, as shown by the fluorescence of NBD-PC, with a characteristic distance between two neighboring aggregates of about 8-15 $\mu \mathrm{m}$ (Figure 2A, bottom). This typical dotted pattern was reproducibly observed on all parts of the interface. Moreover, no fluorescent spot was observed above or below the interface; this shows that the dotted fluorescence pattern corresponds to phospholipid aggregates organized at the interface. To characterize the degree of organization of these phospholipid aggregates, we calculated the $2 \mathrm{D}$ pair-correlation function from the fluorescence image of the interface. Figure 2B shows a typical 2D pair correlation function from an image (488 $\times 488 \mu \mathrm{m})$ obtained $18 \mathrm{~h}$ after preparation of the interface (same conditions as in Figure 2A bottom). It shows that phospholipid aggregates are locally organized with a partial hexagonal order and a characteristic average distance between aggregates of $11 \mu \mathrm{m}$. However, the phospholipid aggregates are rather polydisperse in size, and no order emerges at long distance. Just below the interface, we observed many liposomes, seemingly attached to the interface (Figure 3A inset). At the same time, we found liposomes floating in the water phase as well as settled at the bottom of the observation chamber (Figure 3A). In confocal transmission mode, the inner and outer parts of liposomes look identical (Figure 3B); this shows that all the observed spherical particles in the water phase are liposomes and not water-in-oil droplets. Moreover, by comparing the fluorescence intensity of observed liposomes to that of unilamellar and multilamellar ones obtained by other techniques, we hypothesize that the membranes of the observed liposomes 
were unilamellar or contained only a small number of bilayers. We cannot exclude the possibility that the membrane contains traces of oil.

All the generated liposomes were spherical and presented one typical fluorescent spot (indicated by arrows in Figure 3A) that was attached to the membrane and was assigned to a phospholipid aggregate coming from the interface. In some cases, this phospholipid aggregate was shared between two or three liposomes (liposome multiplex; Figure 3C). In a typical experiment, 100-500 liposomes were obtained with a diameter in the range 1-100 $\mu \mathrm{m}$. Figure 3D shows the distribution of liposome diameters obtained in one particular experiment. In this case, 363 liposomes were obtained with an average diameter of $34.0 \mu \mathrm{m}$. By assuming bilayered membranes and an area per polar head of approximately $0.7 \mathrm{~nm}^{2}$,[10] we could estimate from the size distribution that the total number of phospholipid molecules in the liposome membranes was about $5.7 \times 10^{12}$, which represents approximately $0.08 \%$ of the initial amount of phospholipid dissolved in oil and approximately $26 \%$ of the number of phospholipid molecules necessary to form a monolayer at the oil/water interface. The yield of liposome production with this method is thus comparable to that of natural swelling.

We also studied the effect of temperature and the concentration of phospholipid in the oil. For a temperature less than $20{ }^{\circ} \mathrm{C}$, no vesicles were formed. At $25{ }^{\circ} \mathrm{C}$ and a DOPC concentration $\geq 1 \mathrm{mM}$, the spontaneous formation of liposomes was observed. The properties of the obtained liposomes (size distribution, membrane thickness, presence of a phospholipid spot attached to the membrane) were similar to those of liposomes obtained at a DOPC concentration of $1 \mathrm{mM}$. However, the increase in phospholipid concentration was accompanied by an apparent roughening of the interface, an increase of fluorescence intensity, that is, a larger number of phospholipids at the interface, a larger number of liposome multiplexes, and the formation of about $100 \mu \mathrm{m}$ myelin-like structures at the interface (Figure 4).

We observed that when an interface was formed between oil containing phospholipids and water, phospholipids slowly organized at the interface to form a dotted pattern of phospholipid aggregates that was accompanied by the spontaneous generation of giant liposomes. It is known that phospholipid molecules organize at oil/water interfaces, and various kinds of organization have been described in the literature.[11] At a low concentration, lipid molecules have been reported to organize in a multilayered fashion at the interface; this leads to spontaneous emulsification and 
formation of onion microstructures.[12] In our experiments, when the DOPC concentration was below $1 \mathrm{mM}$, we also observed the formation of regular arrays of oil-in-water microdroplets by a spontaneous emulsification process (see the Supporting Information). However, for a concentration $\geq 1 \mathrm{mM}$, a novel type of organization was observed. Phospholipids form aggregates at the interface that organize slowly to form a dotted pattern, as shown in Figure 2. It is thus interesting to discuss the relationshipbetwe en the formation of this phospholipid pattern and the spontaneous generation of liposomes. At a low phospholipid concentration $(<1 \mathrm{mM})$ and when no phospholipid dotted pattern was observed, no giant liposome was observed in the water phase or at the interface. In contrast, when the phospholipid pattern was formed, that is, for phospholipid concentrations $>1 \mathrm{mM}$, we systematically observed the formation of large number of liposomes at the interface as well as in the bulk water phase, as shown in Figure 3. This shows that the presence of the phospholipid pattern seems to be necessary for liposome formation.

Furthermore, since the typical phospholipid aggregate attached to the liposome membrane in the bulk water phase is comparable in size and shape to that found at the interface, one can hypothesize that it comes from the interface. Moreover, the facts that liposome multiplexes always share the same phospholipid aggregate and that a lot of liposomes are found just below the phospholipid pattern at the interface tend to show that liposomes originate from the phospholipid aggregates at the oil/water interface. Thus, we proposed the following mechanism: 1) at a sufficiently high concentration in the oil phase, phospholipid molecules form aggregates at the oil/water interface; 2) these aggregates reorganize slowly to form a pattern with a local order; 3) liposomes swell in the water phase from these aggregates and eventually detach from the interface. To clarify the molecular and physical origin of this mechanism, we are now performing experiments with different kinds of oil and phospholipids as well as developing methods to characterize in a quantitative manner the organization of phospholipid molecules at the oil/water interface.

In conclusion, we report the emergence of a novel phospholipid organization at oil/water interface accompanied by the spontaneous generation of giant liposomes in the water phase when an interface is made between phospholipid-containing mineral oil and water. The obtained liposomes are spherical and have a size comparable to typical cell dimensions (1-100 $\mu \mathrm{m})$. This phenomenon can be further combined with the possibility of encapsulating lipophilic molecules initially solubilized in the oil phase, or used as a 
very simple method to prepare artificial cells.

\section{Experimental Section}

Materials: 1,2-Dioleoyl-sn-glycero-3-phosphocholine (DOPC) was obtained from Wako Pure Chemicals (Osaka, Japan). Fluorescent phospholipid 1-oleoyl-2-[12-[(7-nitro-2-1,3-benzoxadiazol-4-yl)amino]

dodecanoyl]-sn-glycero-3-phosphocholine (NBD-PC) was from Avanti Polar Lipids (Alabaster, USA). Mineral oil was purchased from Nacalai Tesque (Kyoto, Japan). PDMS was from Dow Corning. Milli-Q deionized ultrapure water was used for all of the experiments.

Preparation of phospholipid-containing oil: DOPC was dissolved in chloroform/methanol (2:1, v/v) at a concentration of $10 \mathrm{mM}$, and a solution of NBD-PC in chloroform was mixed with the DOPC solution at a molar ratio of 1:1000 (NBD-PC/DOPC). This phospholipid solution (33 mL) was poured into a glass test tube with a diameter of $9 \mathrm{~mm}$ (Maruemu, Osaka, Japan), and the organic solvent was evaporated under nitrogen flow to leave a thin film of phospholipid at the bottom of the test tube. The film was subsequently dried under vacuum for ca. $30 \mathrm{~min}$. Mineral oil was then added onto the film in the test tube prior to ultrasonication for $60 \mathrm{~min}$ at $50{ }^{\circ} \mathrm{C}$ and vortex mixing. We checked by $1 \mathrm{H} \mathrm{NMR}$ in $\mathrm{CDCl}_{3}$ that the phospholipid molecules were not degraded during this solubilization proce-dure. The phospholipid solution was used within one day. Final phospholipid concentrations in oil were 1, 2, and 5 mM.

Preparation and observation of the oil/water interface: The observation chamber was made of PDMS with a cylindrical hole (ca. $4 \mathrm{~mm}$ diameter and ca. $5 \mathrm{~mm}$ depth) and bound to a microscope cover glass slide (Matsunami, Osaka, Japan) previously cleaned by being baked for $1 \mathrm{~h}$ at $500{ }^{\circ} \mathrm{C}$. Ultrapure water $(10 \mathrm{~mL})$ was spread at the bottom of the chamber to make a thin layer (ca. $800 \mu \mathrm{m}$ thick) and topped by mineral oil (10 mL) containing phospholipid (ca. $800 \mu \mathrm{m}$ thick). Then the chamber was placed on the temperature-controlled stage of a inverted Axiovert 100 microscope (Zeiss) equipped with a LSM 510 module (Zeiss) and an INU-ZILSG-FO-BW temperature controller (Tokai Hit, Shizuoka, Japan). Under these conditions, we could characterize the evolution over time of the oil phase, the interface, and the water phase by fluorescence and transmission confocal microscopy. We conducted observations with DOPC 
concentrations from 0.5 to $5 \mathrm{mM}$ in oil.

\section{Acknowledgements}

We thank Prof. T. Yoshimura (Mie University), Dr. K. Takiguchi (Nagoya University), and Dr. T. Hamada (Japan Advanced Institute of Science and Technology) for fruitful discussions. This work was supported by the Japan Society for the Promotion of Science (JSPS) under Grant-in-Aid for Creative Scientific Research (Project No. 18GS0421). A.Y. was funded by a Research Fellowship from the JSPS for Young Scientists. 


\section{Figure caption}

Figure 1: Experimental set-up.

Figure 2: A) Confocal fluorescence (left) and transmission (right) microscopy images of the oil/water interface $1 \mathrm{~min}$ (top) and $18 \mathrm{~h}$ (bottom) after its preparation. The phospholipid is DOPC (0.1 mol\% NBD-PC) at a concentration of $1 \mathrm{mM}$ in oil. The fluorescence dotted pattern is attributed to phospholipid aggregates organized at the interface. B) Top: 2D pair correlation function calculated from the microscopy image shown in A (bottom left) but over a wider area $(488 \times 488 \mu \mathrm{m})$. $\mathrm{X}$ and $\mathrm{Y}$ axes give the horizontal and vertical relative distance while the color provides the intensity of the correlation. Bottom: Pair correlation at $\mathrm{Y}=0$ (dashed line in the $2 \mathrm{D}$ correlation function). Scale bars are $100 \mu \mathrm{m}$.

Figure 3: Confocal microscopy images of liposomes spontaneously formed $18 \mathrm{~h}$ after the preparation of the oil/water interface. The phospholipid is DOPC $(0.1 \mathrm{~mol} \%$ NBD-PC) at a concentration of $1 \mathrm{mM}$ in oil. The temperature was kept at $25{ }^{\circ} \mathrm{C}$. A) Confocal fluorescence microscopy image of liposomes settled at the bottom of the observation chamber. Yellow arrows in-dicate fluorescent spots attributed to phospholipid aggregates attached to the membranes of liposomes. Inset: Confocal microscopy image focused slightly below the oil/water interface. White arrows indicate liposomes attached to the interface. The box size is $240 \times 160 \mu \mathrm{m}$. B) Confocal fluorescence (left) and transmission (right) microscopy image of a liposome in the water phase. The black arrow indicates the liposome membrane. C) Confocal microscopy images of multiple liposomes sharing one phospholipid aggregate (indicated by yellow arrows). D) Distribution of liposome diameters in one experimental chamber. In the case of nonspherical objects, such as in the liposome multiplexes shown in $\mathrm{C}$, the value corresponds to the diameter that a circle of the same contour length would have. Scale bars are $50 \mu \mathrm{m}$.

Figure 4: Confocal microscopy observations $18 \mathrm{~h}$ after interface preparation for a DOPC concentration of $5 \mathrm{mM}$ in oil ( $0.1 \mathrm{~mol} \%$ NBD-PC) and a temperature of $25{ }^{\circ} \mathrm{C}$. A) Confocal fluorescence microscopy image focused slightly below the interface with the same sensitivity as that of Figures 2 and 3. White arrows indicate liposomes attached to the interface. B) Confocal fluorescence microscopy image of liposomes settled at the bottom of the observation chamber. C) Same as A at a lower sensitivity. D) 
Same as A in transmission mode. Scale bars are $100 \mu \mathrm{m}$. 


\section{References}

[1] a) S.-i. M. Nomura, Y. Yoshikawa, K. Yoshikawa, O. Dannemuller, S. Chasserot-Golaz, G. Ourisson, Y. Nakatani, ChemBioChem 2001, 2, 457-459; b) V. Noireaux, A. Libchaber, Proc. Natl. Acad. Sci. USA 2004, 101, 17669 - 17674; c) I. A. Chen, K. Salehi-Ashtiani, J. W. Szostak, J. Am. Chem. Soc. 2005, 127, 13213 -13219.

[2] a) A. Karlsson, R. Karlsson, M. Karlsson, A. S. Cans, A. Stromberg, F. Ryttsen, O. Orwar, Nature 2001, 409, 150- 152; b) Giant Vesicles: Perspectives in Supramolecular Chemistry (Eds.: P. L. Luisi, P. Walde), Wiley, Chichester, 2000; c) K. Tsumoto, S-i. M. Nomura, Y. Nakatani, K. Yoshikawa, Langmuir 2001, 17, 7225 -7228; d) S-i. M. Nomura, K. Tsumoto, T. Hamada, K. Akiyoshi, Y. Nakatani, K. Yoshikawa, ChemBioChem 2003, 4, 1172- 1175; e) L. Limozin, A. Roth, E. Sackmann, Phys. Rev. Lett. 2005, 95, 178101; f) M. Honda, K. Takiguchi, S. Ishikawa, H. Hotani, J. Mol. Biol. 1999, 287, 293- 300; g) A. V. Pietrini, P. L. Luisi, ChemBioChem 2004, 5, 1055 1062.

[3] a) J. P. Reeves, R. M. Dowben, J. Cell. Physiol. 1969, 73, 49-60; b) D. Needham, E. Evans, Biochemistry 1988, 27, 8261 - 8269.

[4] N. Oku, R. C. MacDonald, Biochemistry 1983, 22, 855 -863.

[5] a) M. I. Angelova, D. S. Dimitrov, Faraday Discuss. Chem. Soc. 1986, 81, 303311; b) M. I. Angelova, S. SolOau, Ph. MOlOard, J. F. Faucon, P. Bothorel, Prog. Colloid Polym. Sci. 1992, 89, 127 -131.

[6] B. Bauer, M. Davidson, O. Orwar, Langmuir 2006, 22, 9329 -9332.

[7] a) S. Pautot, B. J. Frisken, D. A. Weitz, Langmuir 2003, 19, 2870 -2879; b) A. Yamada, T. Yamanaka, T. Hamada, M. Hase, K. Yoshikawa, D. Baigl, Langmuir 2006, 22, $9824-9828$.

[8] P. S. Dittrich, M. Heule, P. Renaud, A. Manz, Lab Chip 2006, 6, 488-493.

[9] a) M. Hishida, H. Seto, K. Yoshikawa, Chem. Phys. Lett. 2005, 411, 267 - 272; b) M. Hishida, H. Seto, P. Kaewsaiha, H. Matsuoka, K. Yoshikawa, Colloids Surf. A. 2006, 
284-285, 444-447.

[10] J. N. Israelachvili, Intermolecular and Surface Forces, Academic Press, London, 1985.

[11] a) K. Ogino, M. Onishi, J. Colloid Interface Sci. 1981, 83, 18-25; b)W. J. Benton, K. H. Raney, C. A. Miller, J. Colloid Interface Sci. 1986, 110, 363 - 388; c) Y. A. Shchipunov, P. Schmiedel, Langmuir 1996, 12, 6443 -6445.

[12] S. Pautot, B. J. Frisken, J.-X. Cheng, X. S. Xie, D. A. Weitz, Langmuir 2003, 19, 10281 - 10287. 


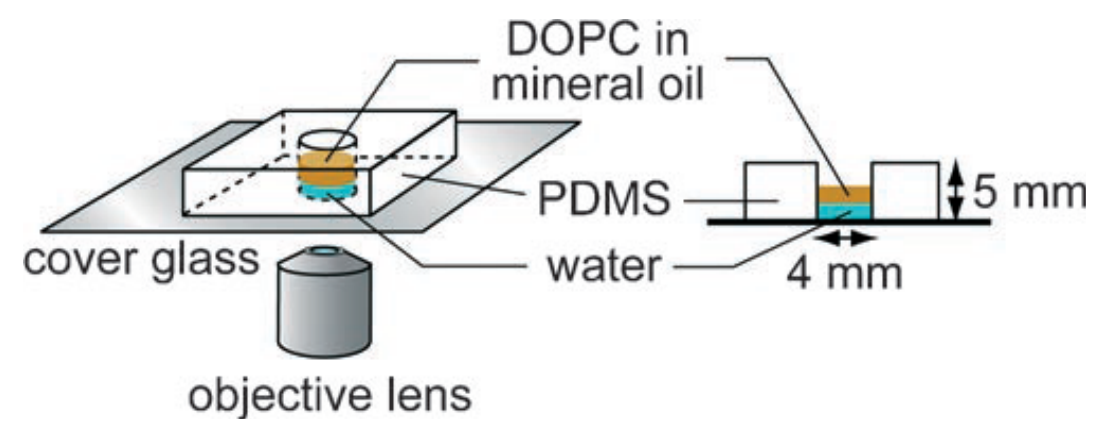

Figure 1 
A)
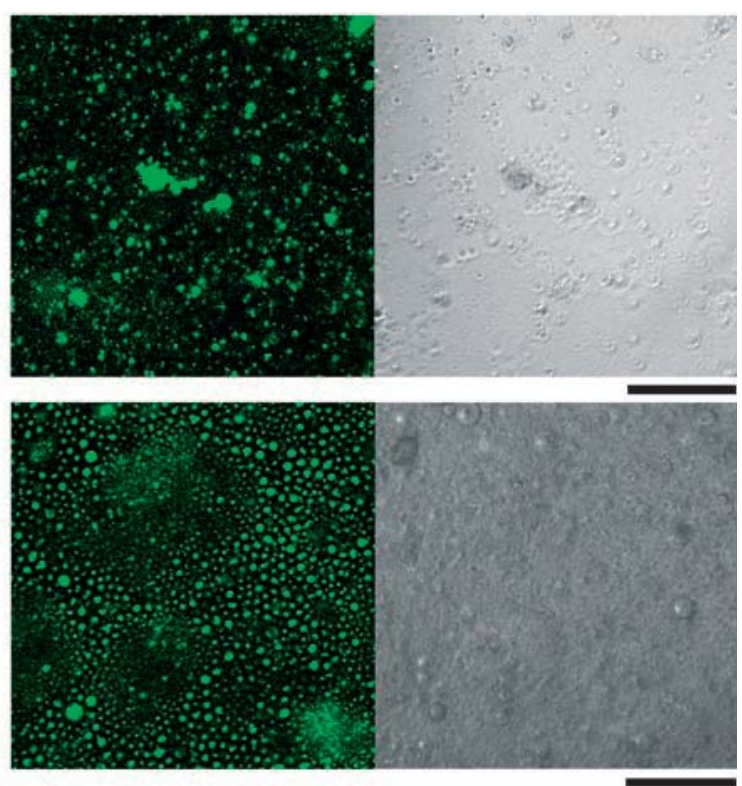

B)

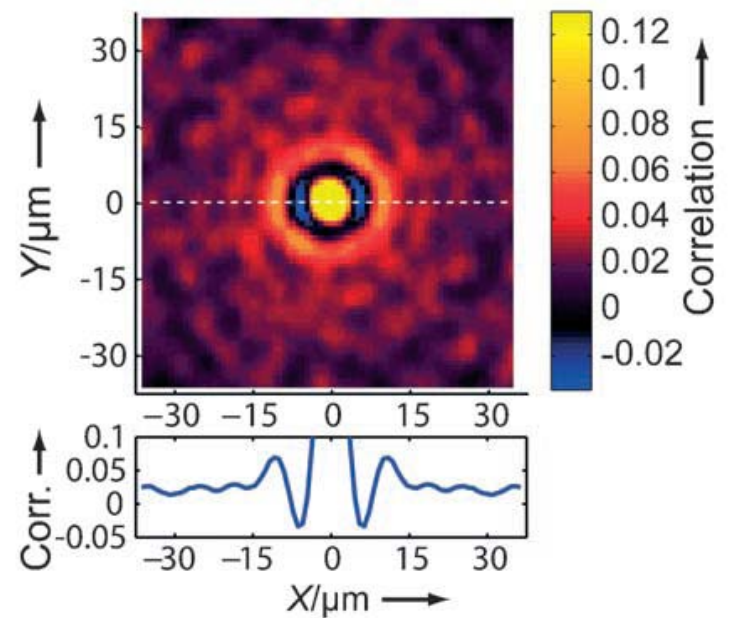

Figure 2 
A)

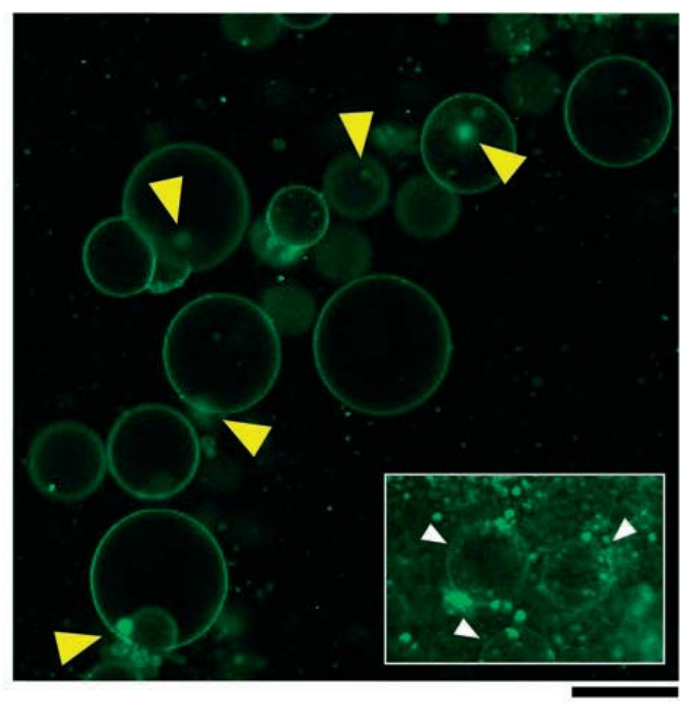

B)
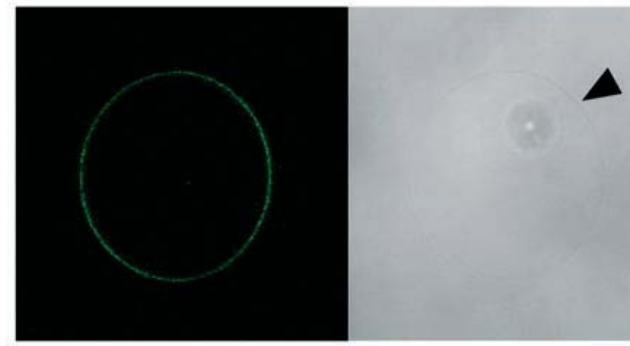

C)
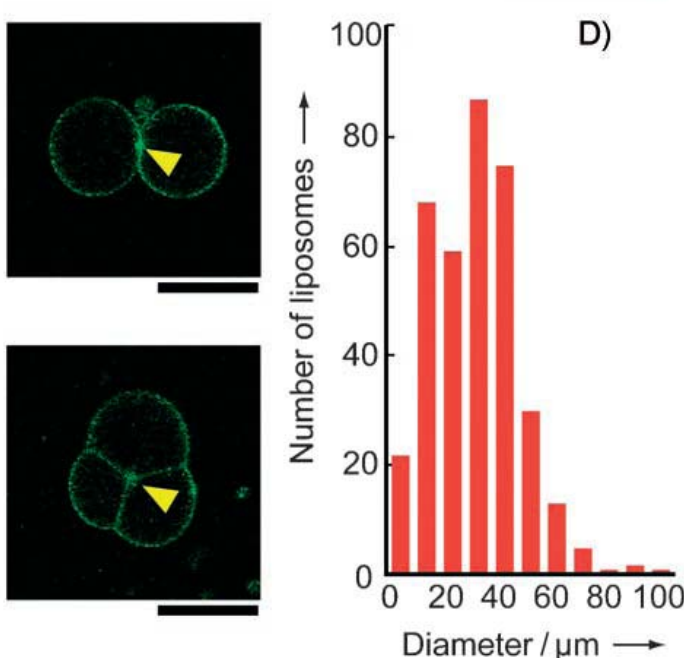

Figure 3 

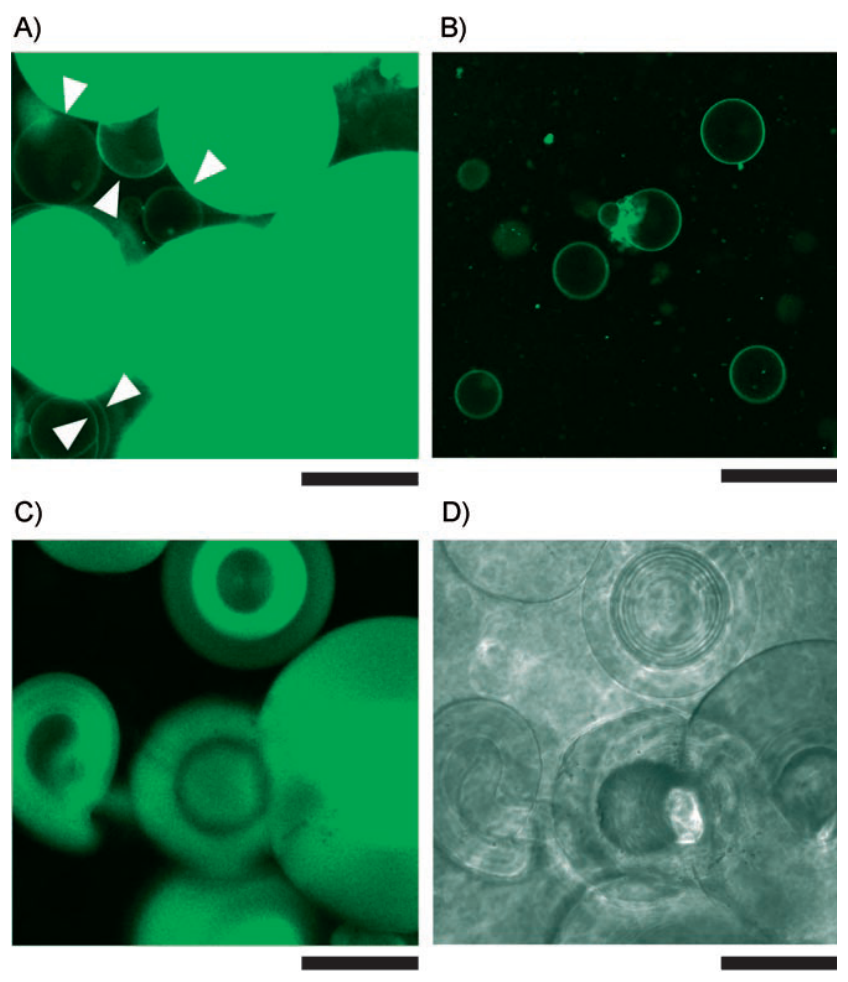

Figure 4 


\section{Supporting information}

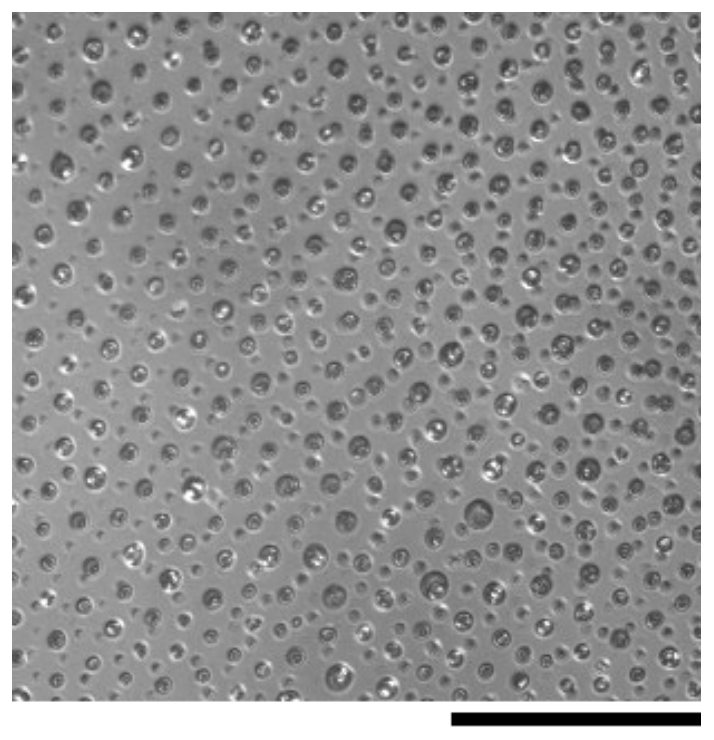

Confocal transmission microscopy image of the oil/water interface 15 hours after its preparation. Phospholipid is DOPC (0.1 mol\% NBD-PC) at a concentration of $0.8 \mathrm{mM}$ in oil. Spontaneously emulsified water-in-oil droplets, visible as dark circles, arranged at the interface into regular arrays, in agreement with previous observations reported in the literature ${ }^{[11]}$. 